\title{
Early-stage growth observations of orientation-controlled vacuum-deposited naphthyl end-capped oligothiophenes
}

\author{
Mathias K. Huss-Hansen $\odot,{ }^{1, *}$ Martin Hodas $\odot,{ }^{2}$ Nada Mrkyvkova $\odot,{ }^{3,4}$ Jakub Hagara, ${ }^{2,3}$ Peter Nadazdy, ${ }^{3}$ \\ Michaela Sojkova $\odot,{ }^{5}$ Simon O. Høegh, ${ }^{6}$ Alina Vlad, ${ }^{7}$ Pallavi Pandit, ${ }^{8}$ Eva Majkova, ${ }^{3,4}$ Peter Siffalovic $\odot,,^{3,4}$ Frank Schreiber, ${ }^{2}$ \\ Jakob Kjelstrup-Hansen (i), ${ }^{6}$ and Matti Knaapila (i) ${ }^{1}$ \\ ${ }^{1}$ Department of Physics, Technical University of Denmark, 2800 Kgs. Lyngby, Denmark \\ ${ }^{2}$ Institut für Angewandte Physik, Universität Tübingen, 72076 Tübingen, Germany \\ ${ }^{3}$ Institute of Physics, Slovak Academy of Sciences, 84511 Bratislava, Slovakia \\ ${ }^{4}$ Center for Advanced Materials Application, Slovak Academy of Sciences, 84511 Bratislava, Slovakia \\ ${ }^{5}$ Institute of Electrical Engineering, Slovak Academy of Sciences, 84104 Bratislava, Slovakia \\ ${ }^{6}$ NanoSYD, Mads Clausen Institute, University of Southern Denmark, 6400 S $\phi$ nderborg, Denmark \\ ${ }^{7}$ Synchrotron SOLEIL, L'Orme des Merisiers, 91190 Saint-Aubin, France \\ ${ }^{8}$ Photon Science, Deutsches Elektronen-Synchrotron (DESY), 22607 Hamburg, Germany
}

(Received 19 October 2020; revised 7 January 2021; accepted 30 April 2021; published 17 May 2021)

\begin{abstract}
We report on the real-time structure formation and growth of two thiophene-based organic semiconductors,

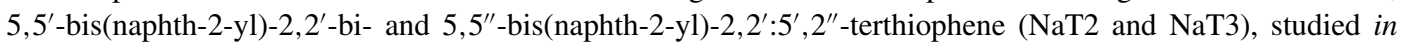
situ during vacuum deposition by grazing-incidence $\mathrm{x}$-ray diffraction and supported by atomic force microscopy and photoabsorption spectroscopy measurements on corresponding ex situ samples. On device-relevant silicon dioxide substrates, for both molecules the growth is observed to transition from two-dimensional (2D) layerby-layer growth to three-dimensional (3D) growth after the formation of a few-molecule-thick wetting layer. The crystal structure of the NaT2 film is considerably more ordered than the NaT3 counterpart, and there is a significant collective change in the unit cell during the initial stage of growth, indicating strain relief from substrate induced strain as the growth transitions from two to three dimensions. In addition, the orientation of the film molecules are controlled by employing substrates of horizontally and vertically oriented few-layer molybdenum disulfide. Both molecules form needle-like crystals on horizontally oriented $\mathrm{MoS}_{2}$ layers, while the NaT3 molecules form tall, isolated islands on vertically oriented $\mathrm{MoS}_{2}$ layers. The molecules are standing on silicon dioxide and on vertically oriented $\mathrm{MoS}_{2}$, but lying flat on horizontally oriented $\mathrm{MoS}_{2}$. These results demonstrate the importance of film-substrate interactions on the thin-film growth and microstructure formation in naphthyl-terminated oligothiophenes.
\end{abstract}

DOI: 10.1103/PhysRevMaterials.5.053402

\section{INTRODUCTION}

Organic semiconductors (OSCs) offer many possibilities as materials in organic electronics, owing to their lowcost, large area processing techniques, and, in particular, the ability to tailor electronic and charge transport properties using synthetic procedures. Applications include organic field effect transistors (OFETs) [1,2], organic photovoltaics (OPVs) [3], organic light-emitting diodes (OLEDs) [4,5], and OFET-based sensors [6,7]. The electronic and optical properties of the OSCs are directly influenced by the microstructure within the organic thin films [8-10], and solving the full complexity of the structure-function relationship remains an important goal in understanding OSC behavior, their processing, and device physics. The devicerelevant thin-film morphology and microstructure differ from bulk and factors such as molecular and macroscopic ori-

\footnotetext{
*Author to whom correspondence should be addressed: mathias.huss-hansen@fysik.dtu.dk
}

entation [11,12], polymorphism including surface-induced phases (SIPs) [13-16], nonequilibrium growth conditions, and interfacial interactions all play important roles. Several methods can be used to investigate the structural properties of OSC films, including x-ray scattering and near edge $\mathrm{x}$-ray absorption fine-structure techniques [17-19], in situ variations thereof [20-23], fluorescence spectroscopy and microscopy [24], Raman spectroscopy [25], optical spectroscopy [26], and electron microscopy [27-29], to name a few.

Dinaphthyl terminated oligothiophenes are OSCs belonging to the extensively studied group of oligothiophenes $[17,19,30]$ and have been shown to display good oxidative and thermal stability, surpassing their alkyl-terminated counterparts. Figure 1 shows the chemical structure of NaTn [31] with $n=2,3$ representing the number of thiophene rings. The two molecules represent two distinct anisotropic cases, with the shorter thiophene dimer having inversion symmetry with respect to its geometric center, while the thiophene trimer has mirror symmetry about the centerline through the middle thiophene ring. Both compounds were found to form 


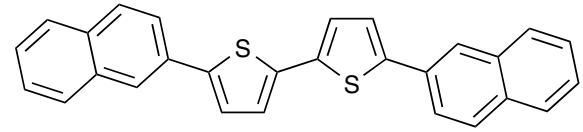

$\mathrm{NaT} 2$

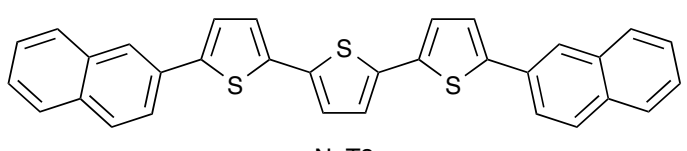

NaT3

FIG. 1. Chemical structures of 5,5'-bis(naphth-2-yl)-2,2'-

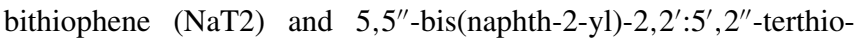
phene (NaT3).

monoclinic crystals in bulk with the molecules packed in the well-known herringbone pattern [31], with NaT3 adopting a three-layer repeat along the long axis, where the molecules in the middle layer are tilted slightly different than the molecules in the other two layers. We recently expanded pioneering single crystal studies of Tian et al. [31] to the film structure and structure formation on various substrates. Molecular mechanics modeling on the two systems in thin-film configuration revealed that $\mathrm{NaT} 3$ form films with significantly greater structural disorder than NaT2 [32], making the two molecules prime candidates for further growth studies. In an earlier article, we reported on the early stage growth of $\mathrm{NaT} 2$ on $\mathrm{SiO}_{2}$ and on chemical vapor deposition (CVD)-grown graphene transferred onto a $\mathrm{Si} / \mathrm{SiO}_{2}$ substrate [33]. We found that the molecular orientation and growth behavior were different in the two cases, but the employed experimental approach did not allow for probing of the critical first few monolayers (MLs). These first few layers are essential from optical and, if the device electrodes are located in the plane, charge-transport point of view. The goal of the present study is to shed light on the fundamental questions of the growth within these first few layers.

In this paper we investigate the early stages of growth in NaT2 and NaT3 films and demonstrate how this growth can be controlled by changing the underlying substrate. Films of NaT2 and NaT3 were prepared by vacuum deposition onto silicon dioxide and onto oriented few-layer molybdenum disulfide $\left(\mathrm{MoS}_{2}\right)$ substrates. The films were studied by real-time in situ grazing incidence $\mathrm{x}$-ray diffraction (GIXRD) experiments during the deposition and complemented by ex situ AFM and UV/Vis absorption experiments. Performing the experiments in real-time and in vacuum conditions ensures no degradation of the films due to ambient exposure and allows us to capture transient structures. Moreover, results from many different film thicknesses can be directly compared since they are obtained under absolutely identical conditions and on the same substrate for a given deposition, which is obviously much more difficult for a series of individual films, considering that the exact conditions will inevitably vary. By exploiting the interactions between $\pi$-conjugated OSCs and distinct substrates, we are able to preferentially align the molecular backbone of our molecules with respect to the surface and identify distinct growth modes. This work builds upon previous characterization studies of NaTn films [32-36] and provides insight into the growth of small-molecule OSC thin films.

\section{EXPERIMENT}

\section{A. Materials and substrates}

The NaT2 and NaT3 molecules (see the chemical structures in Fig. 1) used in this study were synthesized following the Suzuki cross-coupling approach reported in Ref. [37]. The $\mathrm{MoS}_{2}$ layers were prepared by rapid sulfurization of Mo films on $\mathrm{Al}_{2} \mathrm{O}_{3}$ substrates, as described in Ref. [38]. This technique produces crystalline $\mathrm{MoS}_{2}$ layers of high quality. Depending on the thickness of the initial Mo film [39], the resulting $\mathrm{MoS}_{2}$ layers are aligned either parallel to the substrate plane (hereafter referred to as $\|-\mathrm{MoS}_{2}$ ), or perpendicular to the substrate plane (hereafter referred to as $\perp-\mathrm{MoS}_{2}$ ). The resulting film thicknesses of the $\|-\mathrm{MoS}_{2}$ and $\perp-\mathrm{MoS}_{2}$ are 3 and $9 \mathrm{~nm}$, respectively. Commercial $\mathrm{Si}$ substrates, with a $1.5-\mathrm{nm}$-thick native oxide layer, were purchased from Silicon Materials (Germany) and BK7 glass substrates were purchased from UniversityWafer (USA).

\section{B. Grazing incidence $x$-ray scattering}

In situ GIXRD measurements were performed at the PETRA III micro and nanofocus X-ray scattering (P03) beamline [40], and at the SOLEIL Surface(s) and Interface(s) X-ray Scattering (SIXS) beamline. NaTn films were deposited by vacuum sublimation in a custom-built vacuum chamber (base pressure of $4 \times 10^{-8}$ mbar) equipped with a cylindrical beryllium window, allowing the incoming and outgoing $x$-rays to penetrate similar to Ref. [41]. The molecules were evaporated onto the substrate from a Knudsen evaporation cell, and the film thickness was monitored by a quartz crystal microbalance (QCM) inside the deposition chamber. Prior to deposition, the substrates were annealed at $573 \mathrm{~K}$ to desorb any impurities and residual contamination. NaTn was deposited onto the substrate (temperature $323 \mathrm{~K}$ ) at typical evaporation temperatures of $468 \mathrm{~K}$ (NaT2) and $508 \mathrm{~K}$ (NaT3), corresponding to rates of $0.03 \AA / \mathrm{s}$ as monitored by the QCM. 2D GIXRD images were recorded every $20 \mathrm{~s}$ with an attenuated beam to prevent x-ray-induced damage of the films. The lack of beam damage was verified by comparing images of films exposed to prolonged illumination with unexposed films. The x-ray beam energy was $11.6 \mathrm{keV}$ at PETRA III and $12 \mathrm{keV}$ at SOLEIL. The incidence angle was kept between the critical angle of the films and the critical angle of the substrate, typically $0.17^{\circ}$. Scattering images were collected using a Pilatus $300 \mathrm{~K}$ detector (PETRA III) and a Si-XPAD3 detector (SOLEIL), with a sample-to-detector distance (SDD) of $149 \mathrm{~mm}$ and $218 \mathrm{~mm}$, respectively.

Ex situ GIXRD measurements were performed at the PETRA III P03 beamline with an X-ray energy of $12.98 \mathrm{keV}$ and an incidence angle of $0.17^{\circ}$. Images were collected using a LAMBDA detector at a SDD of $159 \mathrm{~mm}$. Data reduction was performed in DAWN [42], with additional analysis performed using self-programmed MATLAB scripts. The x-ray data were normalized to the incoming beam intensity and corrected for standard geometrical contributions. The peak positions were determined by fitting linecuts of the reciprocal space map (RSM) by Gaussian or Lorentzian functions depending on the peak shape. The integrated peak intensity was determined as the area under the peak minus the local background. The 
in-plane and out-of-plane unit cell parameters were determined separately by weighted least-squares minimization of the difference between experimental and calculated $q_{x y}$ and $q_{z}$ positions of selected Bragg peaks. For the full set of equations, please see the work of Merlo et al. [43]. The reported single crystal structure was used as a starting point for the minimization [31]. In the employed experimental geometry, we are essentially resolution-limited due to the width of the beam footprint on the sample [44]. We will therefore refrain from doing Scherrer-type grain-size analysis on the data.

\section{Atomic force microscopy and UV/Vis absorption spectroscopy}

Thin films for ex situ characterization were prepared in the same manner as the in situ samples described above with constant evaporation rates of $0.03 \AA / \mathrm{s}$. The evaporation source shutter was closed once the desired thickness was reached, as determined by QCM readings. Samples on different substrate types were prepared simultaneously, resulting in identical nominal film thicknesses. AFM images were collected in ambient conditions immediately upon removing the samples from vacuum using a Veeco Dimension 3100 operated in tapping mode. Image software GWYDDION was used to flatten and analyze the images [45]. UV/Vis absorption spectra were measured using a Shimadzu UV-2700 spectrometer with an integrating sphere add-on. The absorption curves of the clean substrates were measured separately prior to the thin film deposition and later subtracted from the thin film absorption spectra.

\section{RESULTS AND DISCUSSION}

\section{A. Grazing incidence diffraction}

GIXRD studies of the final films were performed to characterize the crystalline structure. The films were deposited onto $\mathrm{Si}$ substrates with a native oxide layer, and onto few-layer $\mathrm{MoS}_{2}$ surfaces, with the layers oriented either horizontally $(\|)$ or vertically $(\perp)$ on the underlying $\mathrm{Al}_{2} \mathrm{O}_{3}$ substrates. We expect the orientation of the $\mathrm{MoS}_{2}$ layers to influence the molecular orientation of the NaTn films, as has been previously shown for diindenoperylene (DIP) molecules [38]. On $\|-\mathrm{MoS}_{2}$, we expect strong interaction between the molecules and the surface through $\pi-\pi$ and $\mathrm{CH}-\pi$ interactions. On the other hand, $\perp-\mathrm{MoS}_{2}$ layers exhibit a large amount of dangling bonds that are readily oxidized [46]. As a result, the oxidized surface of the $\perp-\mathrm{MoS}_{2}$ is expected to interact weakly with the film molecules.

Figure 2 shows the reciprocal space maps (RSMs) of the final films (thickness varying from 15 to $30 \mathrm{~nm}$ ). The data in Fig. 2(a) show that the shorter NaT2 molecule form highly ordered crystals on silicon dioxide, as indicated by the apparent sharpness of the Bragg reflections. Identical scattering patterns were observed irrespective of the sample rotation around the surface normal, indicating isotropic orientation of the mosaic crystallites in the surface plane, also known as fibertexture. All the Bragg reflections were successfully indexed with a monoclinic unit cell, and by minimizing the difference between experimental and calculated peak positions of the 14 most intense Bragg peaks, the lattice parameters were determined to be $a=20.2 \pm 0.2 \AA, b=6.0 \pm 0.2 \AA, c=$ $8.3 \pm 0.1 \AA$, and $\beta=97.2 \pm 0.2^{\circ}$. The NaT2 molecules are oriented edge-on, which is confirmed by the 100 diffraction peak located perpendicular to the substrate at $q_{z}=0.3 \AA^{-1}$. Reflections along $q_{x y}=0 \AA^{-1}$ are inaccessible in GIXRD due to the curvature of the Ewald sphere [47], yet their tails are still observed due to broadening of the orientation distribution of the crystallites.

By contrast, the RSM of the NaT3 film [Fig. 2(b)] is distinctly different with broad reflections at $q_{x y}=1.33 \AA^{-1}$ and $q_{x y}=1.55 \AA^{-1}$. A full refinement of the lattice parameters is not possible from these data. However, we previously showed that NaT3 films likewise form monoclinic crystals on silicon dioxide [48], with similar in-plane lattice parameters to NaT2 and a longer $a$-axis to accommodate the longer NaT3 molecule. The broadening of the reflections along $q_{z}$ indicates a significant disorder in the out-of-plane structure, which we previously speculated was due to a SIP with a large number of flipped molecules [32]. Several orders of the $h 00$ reflections along $q_{x y}=0 \AA^{-1}$ confirm the edge-on orientation of the molecules.

The RSM of the NaT3 film deposited on few-layer $\|-\mathrm{MoS}_{2}$ [Fig. 2(c)] shows that the molecules are oriented face-on, which is expected considering the strong substrate-molecule interactions at the interface. This is exemplified by the 100 reflection pointing close to parallel to the $q_{x y}$ direction. Considering the 102 reflection pointing along $q_{z}$, we are able to successfully identify all four visible reflections with a monoclinic unit cell. In this configuration, the (102) planes lie parallel to the substrate surface, as indicated on the packing motif in Fig. 2(c). The film crystals exhibit a considerable degree of order, as indicated by the relative sharpness of the 111 reflection. At $q_{z}=1 \AA^{-1}$, an intense reflection originating from the (002) planes of $\mathrm{MoS}_{2}$ is visible, verifying the horizontal orientation of the $\mathrm{MoS}_{2}$ layers relative to the $\mathrm{Al}_{2} \mathrm{O}_{3}$ substrate. NaT2 molecules deposited on few-layer $\|-\mathrm{MoS}_{2}$ likewise adopt face-on orientation (see Fig. S1 in [49]).

The RSM of the NaT3 film prepared on few-layer $\perp-\mathrm{MoS}_{2}$ [Fig. 2(d)] also show $h 00$ reflections consistent with edgeon oriented molecules, but unlike the NaT3 film on silicon dioxide, the reflections along the in-plane direction appear as rods emanating from $q_{z}=0$. Together, these observations suggest a crystal structure where the NaT3 molecules have strong ordering along the stacking ( $a$-axis) direction, but have little to no ordering between the crystallites in the plane. The broadening of the $h 00$ reflections indicates a large distribution in the tilts of long molecular axes with respect to the surface normal.

\section{B. Real-time grazing-incidence diffraction}

We performed in situ GIXRD measurements of the NaT2 and NaT3 films during the deposition process to follow and characterize the crystallographic structure of the thin film during growth. Figure 3(a) shows the unit cell parameters of the NaT2 film on silicon dioxide with increasing nominal film thickness, which determined by deposition time times deposition rate. The unit cell parameters are determined from the peak positions of the 100,111, 102, and 202 reflections. The unit cell changes significantly within 

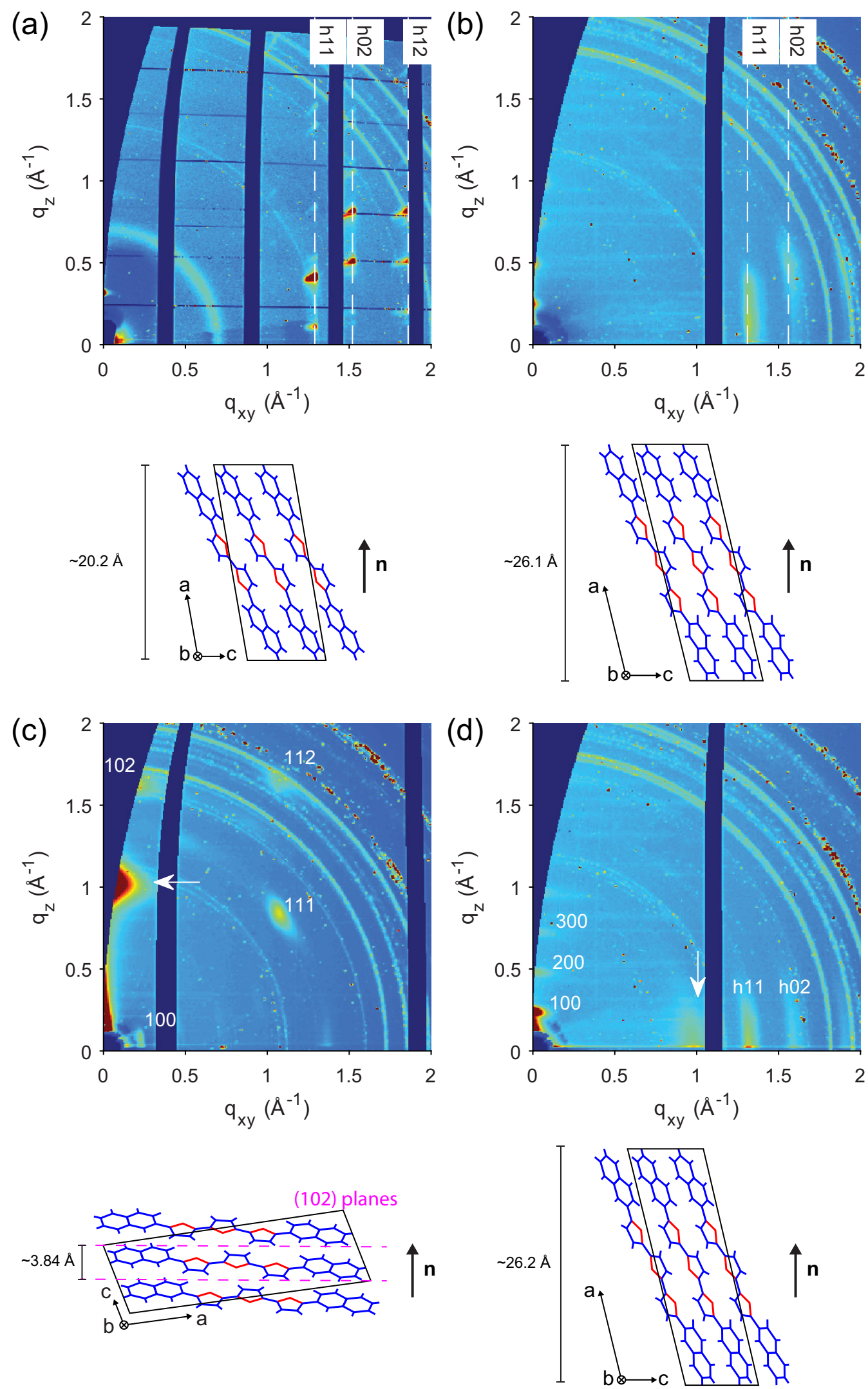

FIG. 2. Two-dimensional RSMs, with proposed packing motifs and approximate layer height, of (a) $\mathrm{NaT}_{2}$ film on $\mathrm{SiO}_{2}$, (b) $\mathrm{NaT}_{3}$ on $\mathrm{SiO}_{2}$, (c) NaT3 on $\|-\mathrm{MoS}_{2}$, and (d) NaT3 on $\perp-\mathrm{MoS}_{2}$. Dashed lines in (a) and (b) indicate diffraction from planes with matching $k$ and $l$ indices, highlighting the 2D in-plane ordering. Arrows in the RSM in (c) and (d) indicate the scattering peaks from the MoS $2(002)$ plane. The isotropic diffraction rings belong to the beryllium entry and exit windows of the deposition chamber (at $q \simeq 0.7 \AA^{-1}, q \simeq 1.3 \AA^{-1}$, and $q>1.7 \AA^{-1}$ ). The surface normal direction is indicated by $\mathbf{n}$ in the packing motifs. 

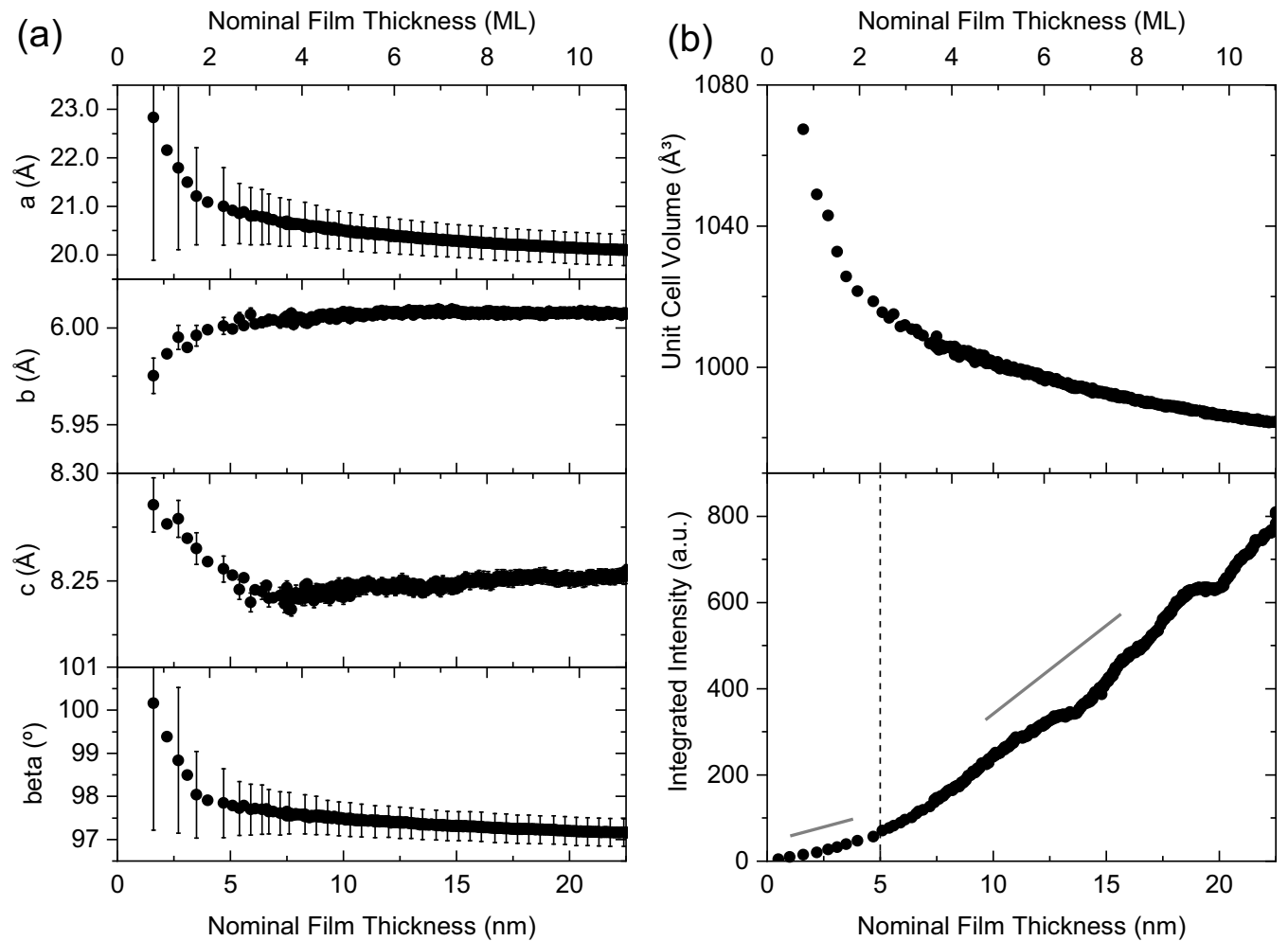

FIG. 3. Real-time GIXRD data of NaT2 deposited on silicon dioxide as a function of nominal film thickness (time $\times$ deposition rate). (a) Unit cell parameters calculated from peak positions of the 100,111, 102, and 202 reflections on the RSM. The secondary abscissa on top denotes the approximate number of molecular layers, given by the average layer thickness during the deposition. (b) Unit cell volume and integrated scattering intensity of the 111 reflection. The dashed vertical line indicates the approximate separation between regions of linear growth with different scaling factors. Solid lines are guides for the eye only.

the first 4 to $6 \mathrm{~nm}$, corresponding approximately to 2 to 3 MLs, both in the plane ( $b$ - and $c$-axes) and out-ofplane ( $a$-axis). We attribute the changes in the in-plane lattice dimensions to strain relief from surface induced strain. After approximately $6 \mathrm{~nm}$, the in-plane lattice parameters remain almost unchanged for larger thicknesses of the film, indicating that the surface-induced strain is fully relieved after $\sim 3$ MLs. On the other hand, the $a$-axis continues to compress throughout the entire deposition. The $a$-axis compression is most pronounced within the first $2 \mathrm{MLs}$, and is accompanied by a $2^{\circ}$ shift in the unit cell tilt. After 2 MLs, the $a$-axis compression and change in $\beta$ slow down but continue until the final thickness of $22 \mathrm{~nm}$ is reached. We attribute the $a$-axis compression and change in $\beta$ to reorganization of the molecules inside the unit cell. The change in unit cell volume is shown in Fig. 3(b), revealing an $8 \%$ decrease in between the initial ML and the final film.

The integrated scattering intensity of the 111 reflection is plotted in Fig. 3(b). For perfect epitaxial layer-by-layer growth [50], the integrated scattering intensity of a Bragg reflection is expected to scale linearly with film thickness. In the case of NaT2 on silicon dioxide, we can distinguish two regions based on the slope of curve: (1) early-stage growth, from 0 to $5 \mathrm{~nm}$, and (2) late-stage growth, which comprises everything above $5 \mathrm{~nm}$. In both regions, the integrated scattering intensity is found to scale approximately linearly with the film thickness, albeit with different scaling factors. We speculate that this is caused by the film molecules undergoing a restructuring and/or reordering in the transition from early-stage to late-stage growth. This change in molecular conformation and/or orientation changes the films structure factor, which results in different electron densities, and therefore scattering intensities, of different layers. There is no evidence of peak splitting in the Bragg reflections, indicating that the structure changes as a whole and not just with the formation of a new layer. This picture is consistent with the observed changes in the unit cell within the first few MLs. A similar change in the electron density within the first MLs was observed for DIP grown on silicon dioxide by Kowarik et al. [51].

Figure 4 shows RSMs of NaT2 films on silicon dioxide measured by ex situ GIXRD with nominal film thicknesses of $4 \mathrm{~nm}$ and $11 \mathrm{~nm}$, thus corresponding to situations from the separate growth domains identified in Fig. 3(b). Ex situ GIXRD measurements allows for longer exposure times and fine-tuning of the incidence angle, resulting in higher signal-to-noise ratio of the RSMs. The 4-nm-thick sample, corresponding nominally to $2 \mathrm{MLs}$, shows Bragg peaks elongated along $q_{z}$ and virtually no $h 00$ reflections, indicative of limited out-of-plane order (often seen in liquid-crystal like films). In comparison, the 11-nm-thick sample, corresponding to five nominal layers, shows many more reflections (including several orders of $h 00$ reflections) pointing to a full, three-dimensional (3D) crystal. The results imply a transition from predominantly two-dimensional (2D) crystals with little 
(a)

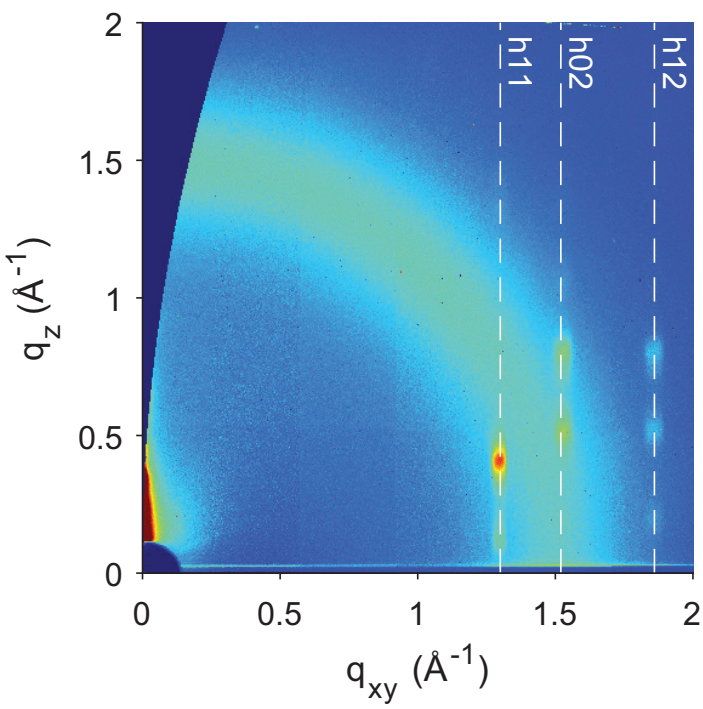

(b)

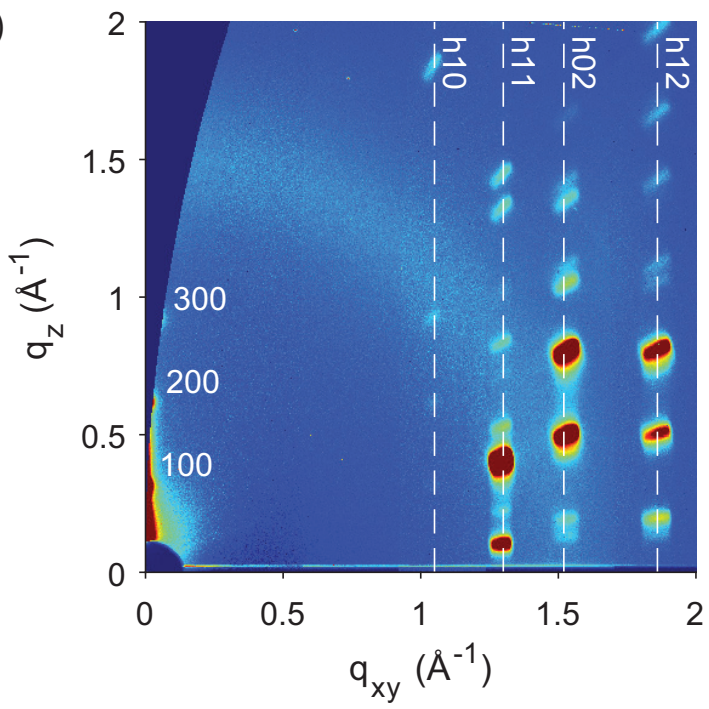

FIG. 4. Ex situ RSMs of (a) $4 \mathrm{~nm}$ and (b) $11 \mathrm{~nm}$ nominally thick NaT2 films prepared on silicon dioxide. The diffuse scattering ring at $q \simeq 1.5 \AA^{-1}$ originates from the substrate. Dashed lines indicate scattering from planes with identical $k$ and $l$ indices.

out-of-plane order, to fully 3D crystals with high in-plane and out-of-plane order. This transition coincides with the transition from early-stage growth to late-stage growth on Fig. 3(b). However, we emphasize that the experiment is not identical to the above-described in situ GIXRD measurements, and that the films have been exposed to ambient conditions prior to measurements.

For the NaT3 film deposited on silicon dioxide, it is not possible to refine the full unit cell from the RSM, but we can still identify the essential structural characteristics with increasing film thickness. Figure 5(a) shows the time evolution of the $d_{100^{-}}$and ( $d_{h 11^{-}}$-spacings, measured along the substrate normal and substrate plane, respectively. The values are calculated from $2 \pi / q$, where $q$ is the peak position of the 200 and $h 11$ reflections on the RSM. The shaded area indicates the cutoff before which peak fitting is impossible due to overlap between the 200 reflection and the off-specular diffuse scattering from the surface. Once again we observe a
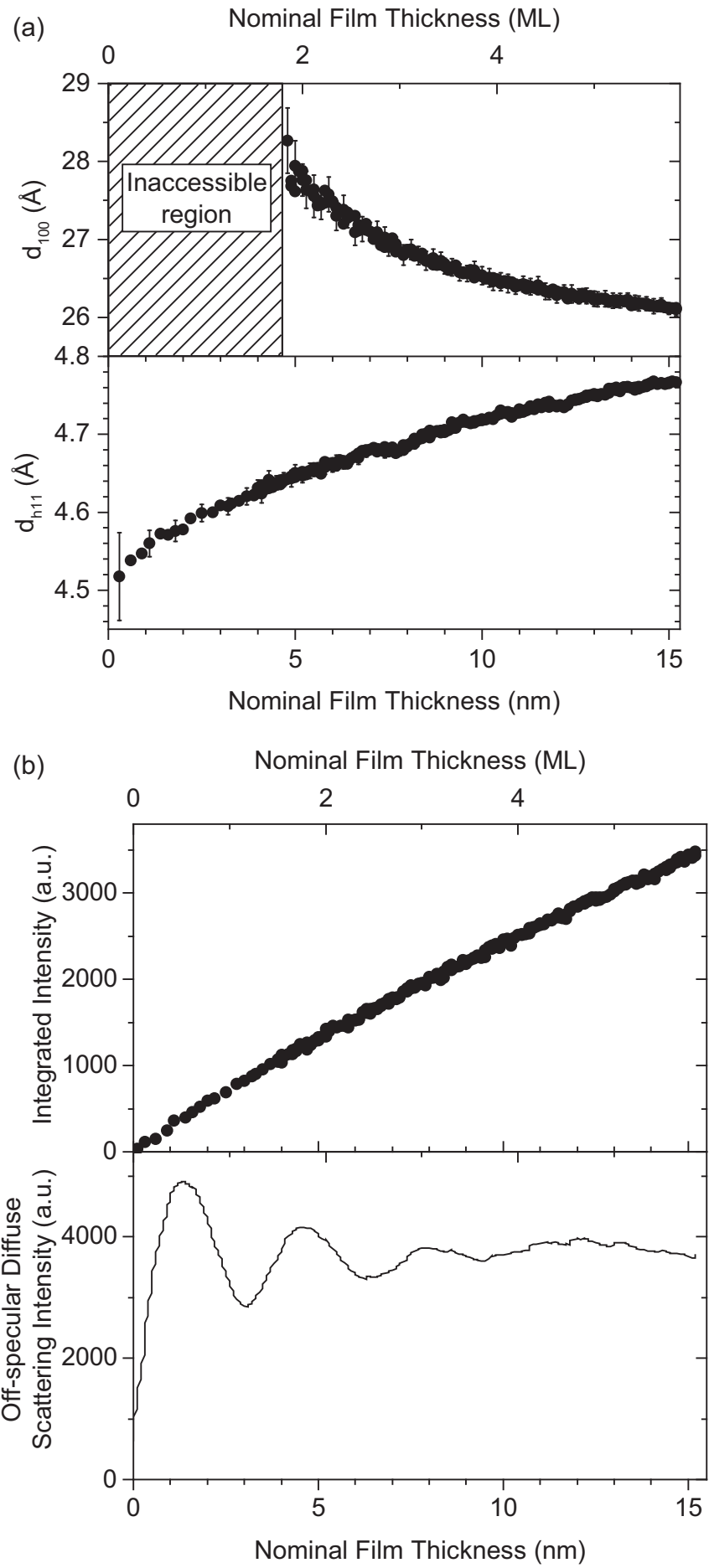

FIG. 5. Real-time GIXRD data of NaT3 deposited on $\mathrm{SiO}_{2}$ as a function of nominal film thickness. (a) Evolution of out-of-plane and in-plane lattice parameters. The inaccessible region is due to overlap between the 200 reflection and off-specular diffuse scattering. (b) Integrated scattering intensity of the $h 11$ reflection and off-specular diffuse scattering (at $q_{x y}=0-0.04 \AA^{-1}$ and $q_{z}=0.13-0.18 \AA^{-1}$ ) with increasing film thickness.

significant compression of the out-of-plane component of the unit cell, with the value seemingly starting to plateau after 5 MLs. The unit cell expands by almost $0.1 \AA$ along the [h11] direction within the first $2 \mathrm{~nm}$, after which it continues 
to expand at a relatively constant albeit slower rate until the final film thickness is reached. These findings point towards a greater surface induced strain in the NaT3 films, as compared with the shorter molecule NaT2 films.

Figure 5(b) shows the integrated scattering intensity of the $h 11$ reflection together with the off-specular diffuse scattering from the area just above the beamstop on the RSM $\left(q_{x y}=0-\right.$ $0.04 \AA^{-1}$ and $\left.q_{z}=0.13-0.18 \AA^{-1}\right)$. From the figure, it is evident that the integrated scattering intensity increases linearly with the film thickness, in contrast to the NaT2 film, where two regions with different scaling could be identified. The offspecular diffuse scattering, which is known to be connected to surface roughness [52], can be used to gain information about the film growth. For perfect layer-by-layer growth, the off-specular diffuse scattering is expected to oscillate with a period of $1 \mathrm{ML}$. As the first layer starts to form on the surface, the surface roughness, and therefore the intensity of the diffuse scattering, will rise due to the formation of islands. The surface roughness (and therefore the diffuse scattering) will reach its maximum at around $0.5 \mathrm{ML}$, corresponding to half surface coverage. As the islands starts to coalesce, the surface roughness and diffuse scattering will consequently start to decrease, reaching a minimum at exactly 1 ML (i.e., a perfectly smooth film with complete surface coverage). The same pattern would be expected for the formation of the second layer and third layer and so on, manifesting as oscillations in the diffusely scattered intensity with matching amplitude and a periodicity of $1 \mathrm{ML}$. An example of such oscillations in the diffusely scattered intensity in layer-by-layer growth can be found for the isotropic fullerene C60 in the work of Bommel et al. [53]. The diffuse scattering intensity on Fig. 5(b), however, does not follow the pattern expected for perfect layer-by-layer growth. Instead, the oscillation period is slightly larger than $1 \mathrm{ML}(1.2 \mathrm{ML})$ for the first oscillation, and the amplitude is seemingly damped with each subsequent oscillation. The attenuation of the diffuse scattering and the oscillation period not exactly matching $1 \mathrm{ML}$ points towards imperfect layer-by-layer growth of the NaT3 film. Similar findings for the off-specular diffuse scattering intensity was reported for imperfect layer-by-layer growth of $\alpha, \omega$-hexyldistyryl thieno thiophene (DH-DSTT) [54], which by nature is closer to our system than the fullerene C60 model system. The lack of oscillations after $3 \mathrm{ML}$ in the diffuse scattering intensity suggest that the growth has fully transitioned from imperfect layer-by-layer growth to 3D island growth (vide infra).

Finally, we consider the case of NaT3 films deposited on oriented $\mathrm{MoS}_{2}$ surfaces. Figure 6 shows the lattice spacings as a function of nominal film thickness (i.e., measured by the QCM) for NaT3 deposited on both $\|-\mathrm{MoS}_{2}$ and $\perp-\mathrm{MoS}_{2}$. For the NaT3 film deposited on $\perp-\mathrm{MoS}_{2}$, the compression of the $d_{100}$-spacing is similar to the what was observed for NaT3 on silicon dioxide, while the $d_{h 11}$-spacing is approximately $3 \%$ larger at a film thickness of $0.9 \mathrm{~nm}(4.69 \AA$ compared to $4.55 \AA$ on silicon dioxide). This could be an indication of a slightly better lattice match between the NaT3 thin film and the $\mathrm{MoS}_{2}$. An increase in the [h11] direction of the lattice is also observed on the $\perp-\mathrm{MoS}_{2}$ with increasing film thickness, reaching a final value of $4.81 \AA$ at $15.3 \mathrm{~nm}$. As previously shown, the NaT3 molecules deposited on $\|-\mathrm{MoS}_{2}$ adopt face-

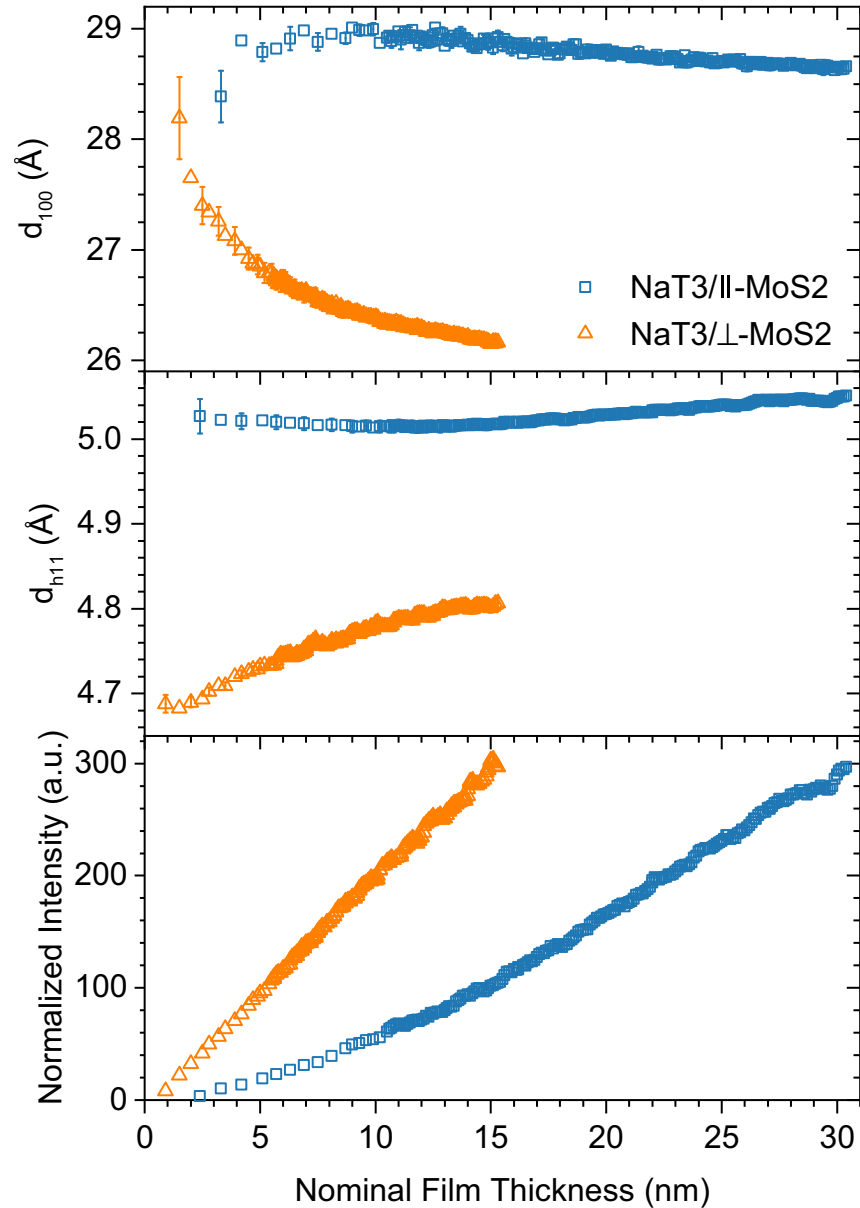

FIG. 6. Lattice parameters and integrated scattering intensity of the $h 11$ reflection for NaT3 molecules with face-on orientation on $\|-\mathrm{MoS}_{2}$ (blue squares) and edge-on orientation on $\perp-\mathrm{MoS}_{2}$ (orange triangles) with increasing nominal film thickness. The intensity values are normalized to the final intensity value.

on configuration. The unit cell of the crystalline face-on phase is significantly larger than the unit cell of the crystalline edgeon phase found growing on $\perp-\mathrm{MoS}_{2}$, clearly evident by the larger $d_{100^{-}}$and $d_{h 11^{-}}$-spacings shown in Fig. 6. For NaT3 deposited on $\|-\mathrm{MoS}_{2}$, the $d_{100}$-spacing first expands slightly and, after certain film thickness is reached, starts to compress at a slower albeit constant rate. A similar evolution of the (100) lattice plane spacing was observed for the thiophene-based molecule 1-[5-(2-naphthyl)-2,2-bithiophen-5-yl]hexan-1-one (NCOH) [55]. The $d_{h 11}$-spacing exhibits the opposite behavior, first undergoing a small compression followed by a slow expansion. The $h 11$ integrated scattering intensity increases linearly with the nominal film thickness for NaT3 deposited on $\perp-\mathrm{MoS}_{2}$, For the NaT3 film deposited on $\|-\mathrm{MoS}_{2}$, the integrated scattering intensity of the $h 11$ reflection scales nonlinearly with effective film thickness.

\section{Atomic force microscopy}

To characterize the surface morphologies of the thin films, we used ex situ AFM on samples at various stages of film growth. We prepared vacuum-deposited films with nominal thicknesses of $2.0 \mathrm{~nm}, 4.8 \mathrm{~nm}$, and $14.9 \mathrm{~nm}$, respectively, by 

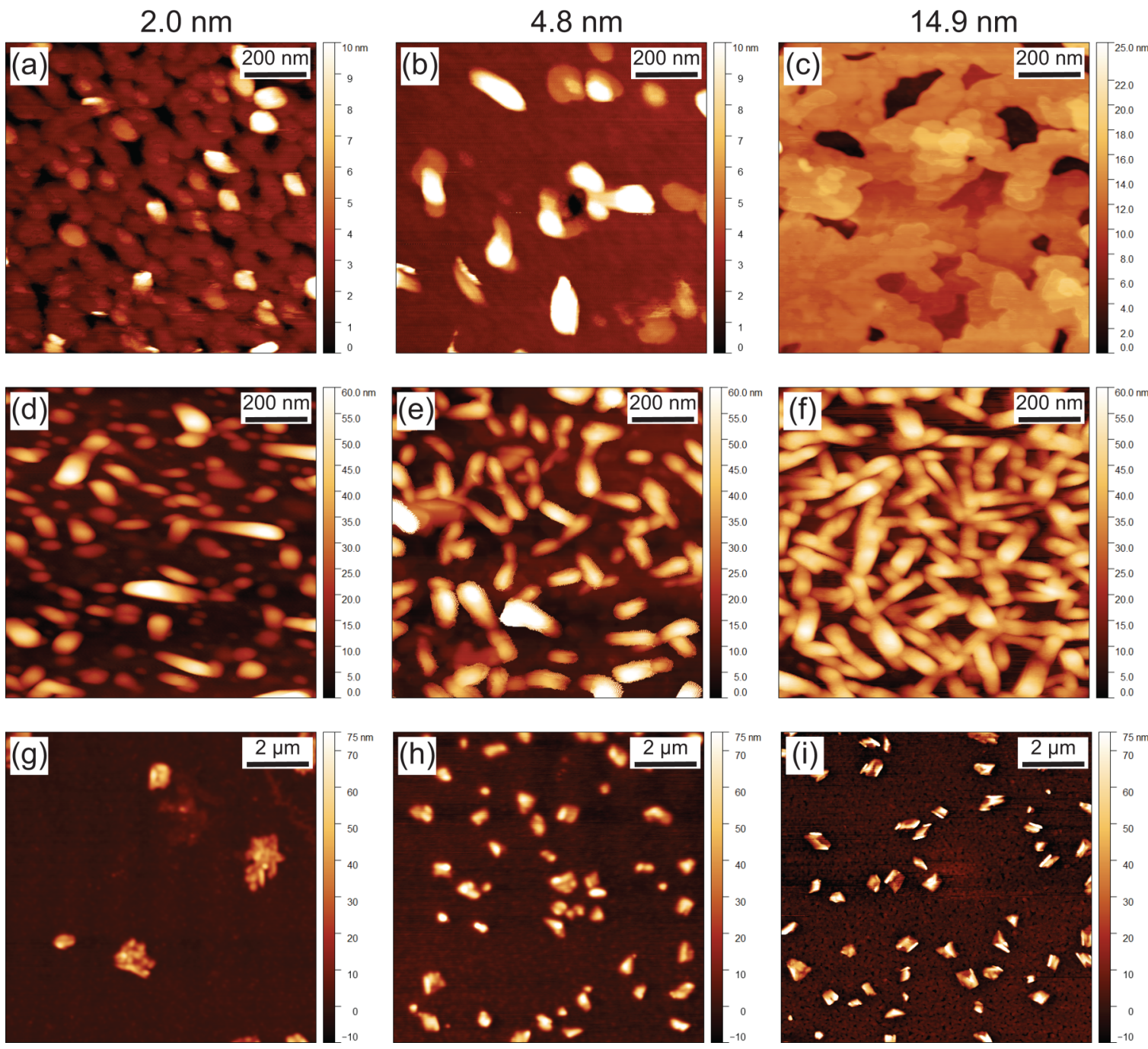

FIG. 7. AFM images of vacuum deposited NaT3 films with different nominal thicknesses, as determined by corresponding QCM readings, on (a)-(c) silicon dioxide, (d)-(f) $\|-\mathrm{MoS}_{2}$, and (g)-(i) $\perp-\mathrm{MoS}_{2}$.

closing the deposition shutter at corresponding readings of the QCM. Figure 7 shows the AFM results for NaT3 films deposited on all three types of substrates. On silicon dioxide [Fig. 7(a)], the sample with a nominal film thickness of $2.0 \mathrm{~nm}$, corresponding to $0.8 \mathrm{MLs}$, displays a morphology of connected islands that form a near complete ML. A few islands are observed on top of the first ML, indicating the nucleation of the second and third layer. The second image [Fig. 7(b)], corresponding to the sample with a nominal film thickness of $4.8 \mathrm{~nm}$, shows a completed first layer with taller islands on top that range in height from $2.5 \mathrm{~nm}(1 \mathrm{ML})$ to $15 \mathrm{~nm}$ (6 ML). The final sample [Fig. 7(c)] displays a film morphology comprised of repeated 3D island formations, with individual islands exceeding $25 \mathrm{~nm}$ in height. Figure S2 in Ref. [49] shows the corresponding height distributions of the AFM data, where clearly distinguishable peaks can be identified and attributed to the silicon dioxide substrate, and the first and second ML of the NaT3 film, respectively. The NaT2 film on silicon dioxide follows a similar morphological progression (see Fig. S3 in Ref. [49]).
The AFM images of samples with NaT3 deposited on $\|-\mathrm{MoS}_{2}$ [Figs. 7(d) to 7(f)] show that the NaT3 molecules form thin needle-like crystals with heights up to $\sim 60 \mathrm{~nm}$. The surface coverage of the needles increases with increasing nominal film thickness, progressing from isolated needles at $2.0 \mathrm{~nm}$ to a network of interconnect crystals at $14.9 \mathrm{~nm}$. However, the maximum height of the needles does not appear to change with increased film coverage. The film morphology of NaT2 on $\|-\mathrm{MoS}_{2}$ is shown on Fig. S4 in Ref. [49].

Lastly, the AFM image sequence of NaT3 deposited on $\perp-\mathrm{MoS}_{2}[$ Figs. $7(\mathrm{~g})$ to 7 (i)] reveals that the NaT3 molecules form tall, isolated islands on the $\perp-\mathrm{MoS}_{2}$ surface. From the image of the first sample [Fig. 7(g)], with a nominal film thickness of $2 \mathrm{~nm}$, it is evident that the islands are comprised of tall pillar formations, some of which exceeds $60 \mathrm{~nm}$ in height. From Figs. 7(h) and 7(i), it appears that the number of islands is increasing with the amount of material deposited on the substrate, while the individual island morphology remains unchanged. The surface coverage is mostly incomplete for all three samples. 


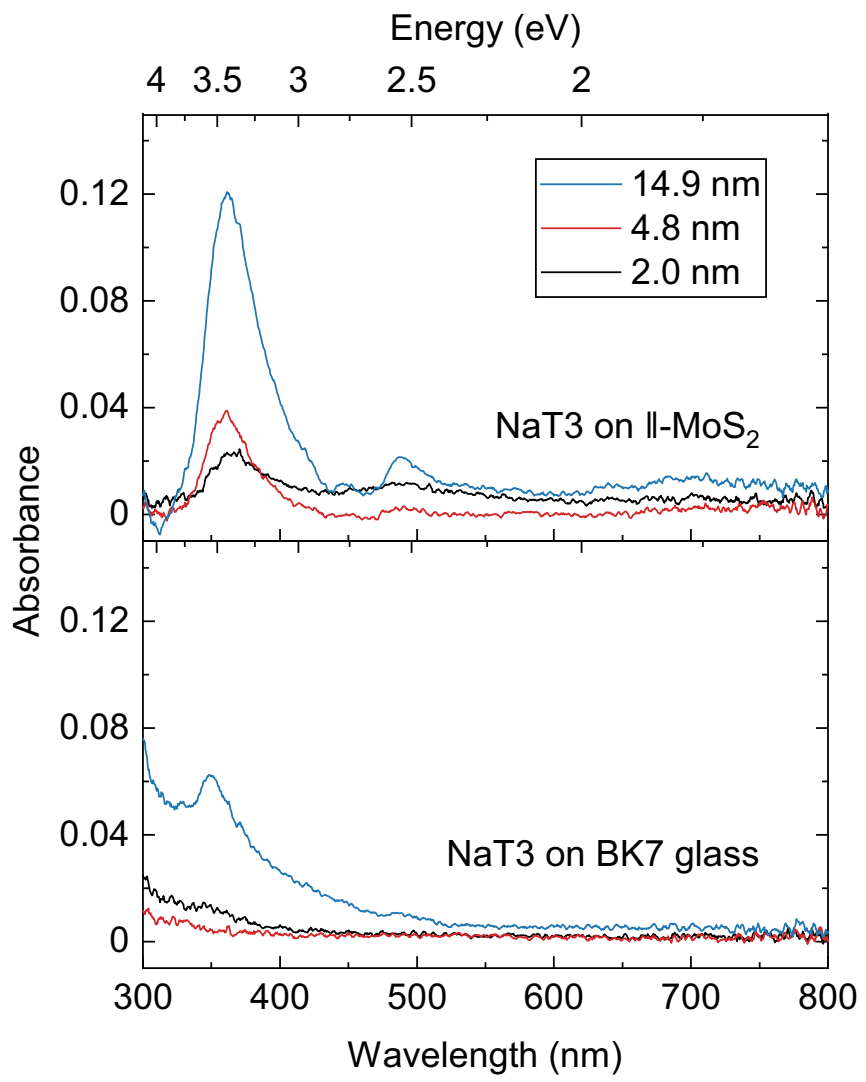

FIG. 8. UV-Vis absorption spectra of NaT3 films with different nominal film thicknesses deposited on $\|-\mathrm{MoS}_{2}$ and BK7 optical glass, corresponding the face-on and edge-on molecular configuration, respectively.

\section{UV-Vis absorption spectroscopy}

Figure 8 shows the UV-Vis absorption spectra measured for NaT3 films of different thicknesses deposited on $\|-\mathrm{MoS}_{2}$. Corresponding data for NaT3 on BK7 optical glass are presented for comparison. These cases represent the face-on on and edge-on configurations of the NaT3 molecules, respectively. The absorption of the pure NaT3 film was obtained by separately measuring and subtracting the substrate absorption curve. The spectra prior to the background subtraction are shown in Fig. S5 in Ref. [49]. The UV-Vis data show an apparent increase of the absorbance with increasing film thickness for both face-on and edge-on oriented NaT3 molecules, with the absorbance being significantly higher for the face-on oriented molecules at the same nominal film thickness. This is expected since the transition dipole moment $\mu$ of NaT3 is aligned parallel to its molecular long axis. In the experimental configuration, the incident light is perpendicular to the surface, while the electric field $E$ is within the surface plane. Thus the absorption is maximized when $\mu$ and $E$ are aligned [56], which is the case for the face-on oriented molecules. Similar results were obtained for diindenoperylene (DIP) molecules on $\|-\mathrm{MoS}_{2}$ [38], and for other small molecules in face-on configurations $[57,58]$.

The absorption spectra of the face-on oriented molecules exhibits clear maxima at $364-358 \mathrm{~nm}$, corresponding to the $\pi-\pi^{*}$ transition, in order of increasing film thickness. A blueshift of the absorption maximum is observed, which is most prominent from $2 \mathrm{~nm}$ to $4.8 \mathrm{~nm}$ nominal film thickness. We suspect that the blueshift of the absorption maximum is caused by a change in the molecular conformation, especially considering the change in the lattice $a$-parameter within the first few deposited layers (Fig. 6). This result is interesting since one would expect the absorption peak to be redshifted with increased film thickness due to dielectric screening effects with increasing number of molecular neighbors $[59,60]$. In comparison, the absorption maximum of the edge-on oriented molecules is at $350 \mathrm{~nm}$. Again, we attribute the shift in transition energy between the two configurations to changes in the molecular conformation. We point out that the absorption spectrum in the edge-on configuration is mostly in agreement with previous reports, with a slight shift of the absorption maximum [31]. We speculate that this due to slight changes in molecular conformation in the very thin films measured here.

\section{E. Growth discussion}

From the real-time GIXRD data and the ex situ AFM images, we can infer certain characteristics of the thin film growth. Figure 9(a) illustrates the suggested growth of NaT2 and NaT3 on silicon dioxide. The two molecules grow in a similar manner with a few notable differences. Both films form crystals with edge-on oriented molecules, but the NaT2 film is more ordered than the NaT3 counterpart. The surface morphology indicates that the NaT2 molecules initially form a ML that wets the substrate, and that this wetting layer is not fully completed before the nucleation onset of the second ML. The second and third MLs are then formed by epitaxial growth, and once again the third layer nucleates before the completion of the second layer. The imperfect layer-by-layer growth observed in the first 2 to 3 MLs is then followed by a transition to $3 \mathrm{D}$ island growth. This behavior is typical for Stranski-Krastanov (SK) growth, where 2D layer-by-layer growth of a wetting layer is followed by $3 \mathrm{D}$ growth at a system-dependent threshold thickness. Moreover, the GIXRD measurements indicate that the unit cell structure of the first few layers undergoes a collective change, with further indications that the molecules inside the unit cell undergoes a restructuring and/or reordering.

The picture is very similar for NaT3 grown on silicon dioxide. The off-specular diffuse scattering and the surface morphology both indicate that the first ML is formed by imperfect layer-by-layer growth, i.e., the onset of the second layer nucleation occurs before the completion of the first layer. However, while the observed morphology indicates that the molecules start to form 3D islands already on top of the first ML, suggesting that the substrate-wetting layer has a thickness of $1 \mathrm{ML}$, the oscillations of the diffuse scattering intensity indicate that at least 2 MLs are formed by imperfect layer-by-layer growth before the growth has fully transitioned to $3 \mathrm{D}$ islands growth. We speculate that the discrepancy between these two results might be due to the real-time GIXRD measurements better capturing the kinetics of the growth, and effects such as postgrowth dewetting [24,26,61] are evidently not seen.

SK growth is often observed in other systems of rod-like small-molecules, including other molecules from the family of oligothiophenes such as $\alpha$-sexithiophene 
(a)

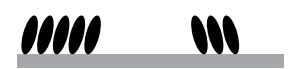

(b)

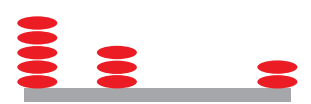

(c)

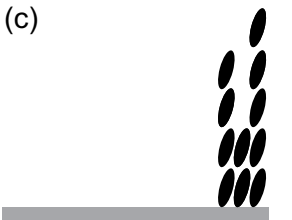

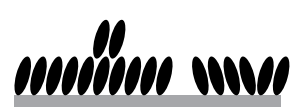
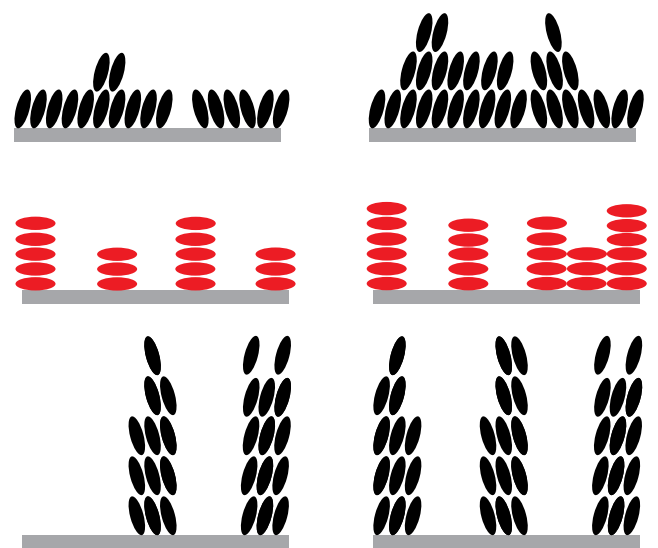
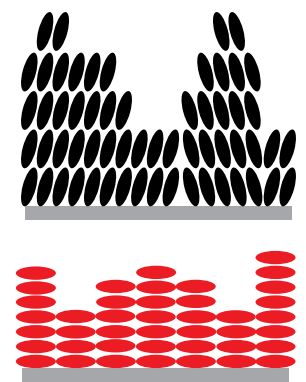

Edge-on molecules
Face-on molecules

Time

FIG. 9. Schematic of the different growth scenarios reported. (a) NaT2 and NaT3 deposited on $\mathrm{SiO}_{2}$ with edge-on oriented growth and initial growth in closed layers, followed by islanding [typically referred to as Stranski-Krastanov (SK) growth]. (b) NaT3 deposited on $\|-\mathrm{MoS}_{2}$ with face-on oriented molecules and islanding [typically referred to as Volmer-Weber (VW) growth]. (c) NaT3 deposited on $\perp$-MoS 2 with edge-on oriented molecules and Volmer-Weber type growth.

$(\alpha-6 \mathrm{~T})$ [23], which has been shown to form a 2-ML-thick substrate-wetting layer on glass substrates before 3D growth sets in. In comparison, DIP on silicon dioxide exhibits layer-by-layer growth for seven monolayers before 3D growth sets in [20]. The transition from 2D to 3D growth in DIP (also referred to as rapid roughening) has been attributed to spatial inhomogeneities, that arise at the boundaries between coalesced islands in the first ML due to different in-plane orientation of the molecular tilt vector [62]. Furthermore, the transition from $2 \mathrm{D}$ to $3 \mathrm{D}$ growth is temperature dependent, as demonstrated for the model system 3,4,9,10-perylene-tetracarboxylic dianhydride (PTCDA) in the work of Krause et al. [63].

Figure 9(b) illustrates the growth of NaT3 deposited on $\|-\mathrm{MoS}_{2}$. The growth is dominated by substrate-molecule interactions, and this leads to nucleation of needle-like crystal structures, that grow as isolated islands (Volmer-Weber-type growth) with the molecules oriented face-on on the substrate. The surface morphology indicate that the fibers nucleate immediately upon the substrate without forming an initial wetting layer, and that the needle density is proportional to the amount of material deposited on the substrate. Considering that the needle height does not appear to exceed $60 \mathrm{~nm}$ as the density increases, we suspect that the growth is thermodynamically limited, and that the observed needle shape is energetically favored under these conditions. The change in lattice parameters indicate a slow expansion of the molecular molecular long axis (situated along the [100] direction of the crystal) followed by a slow compression, combined with a compression along the [h11] direction of the crystal followed by an expansion. Interestingly, the turnover point in the GIXRD data might correspond to the nominal film thickness at which the needles start to coalesce. Needle growth is commonly observed for organic semiconductors on strongly interacting templated substrates [24,64,65], and has previously been demonstrated for NaT3 on muscovite mica [37]. Furthermore, DIP molecules deposited onto sim- ilar $\|-\mathrm{MoS}_{2}$ substrates have been shown to form needle-like crystals, where the average needle height appear to increase with increasing nominal film thickness [38].

Figure 9(c) illustrates the growth of NaT3 on $\perp-\mathrm{MoS}_{2}$, where the molecules are standing edge-on on the surface. Edge-on orientation of the molecules points towards weaker substrate-molecule interactions than for $\|-\mathrm{MoS}_{2}$, presumably because the $\perp-\mathrm{MoS}_{2}$ exhibit chemically active dangling bonds that are readily oxidized [46], leading to an oxidized surface, which is expected to interact weaker with the deposited molecules. The surface morphology images reveal Volmer-Weber-type growth, with tall isolated islands. A low nucleation density of the islands suggests a high nucleation barrier on the $\perp-\mathrm{MoS}_{2}$ substrate, and the lack of in-plane correlation agrees well with the GIXRD data. Furthermore, the surface morphology indicates that the average island size remains relatively constant with increasing nominal thickness, and we therefore assume a linear increase in islands density with increasing nominal thickness based on the integrated scattering intensity. These findings are surprising since the aforementioned DIP molecules grow in accordance to the SK-growth mode on a similar substrate [38].

\section{CONCLUSION}

We investigated the molecular structure and growth of vacuum deposited NaT2 and NaT3 on silicon dioxide and vertically and horizontally aligned few-layer $\mathrm{MoS}_{2}$, using real-time in situ GIXRD and complemented these measurements by AFM and UV/Vis absorption spectroscopy. On device-relevant silicon dioxide, we found that the NaT2 unit cell undergoes collective in-plane changes within the first three molecular layers, indicating that surface-induced strain is relieved beyond this point. This was accompanied by a significant compression in the out-of-plane direction (along molecular long axis), which continued, albeit at a slower rate, until the final film thickness $(22 \mathrm{~nm})$ was reached. The NaT3 
film on silicon dioxide underwent changes in the in-plane and out-of-plane lattice parameters throughout all of the deposition ( 0 to $15 \mathrm{~nm}$ ). The changes in the in-plane unit cell dimensions are greater compared to NaT2, and indicating a grater surface-induced strain compared to NaT2, which is not fully relieved after $6 \mathrm{ML}$. Both films were found to follow Stranski-Krastanov-type growth, where 2D layer-by-layer growth is followed by $3 \mathrm{D}$ islands growth. Neither molecule exhibited perfect layer growth of the first, substrate-wetting ML. In the case of NaT3, the growth was fully transitioned to 3D islands growth after no more than 3 MLs.

By employing vertically and horizontally aligned few-layer $\mathrm{MoS}_{2}$ substrates, the orientation of the molecules could be controlled during the growth. On $\|-\mathrm{MoS}_{2}$, both NaT2 and NaT3 molecules adopted face-on orientation, while NaT3 adopted edge-on orientation on $\perp-\mathrm{MoS}_{2}$. In both cases, the NaT3 unit cell underwent changes in its $d_{100^{-}}$and $d_{h 11^{-}}$ spacings during the deposition, with the largest changes observed during the initial stages of the film formation. Direct images of the surface morphology revealed that both NaT2 and NaT3 molecules formed needle-like crystals on $\|-\mathrm{MoS}_{2}$, while the NaT3 molecules formed isolated islands consisting of tall pillars on $\perp-\mathrm{MoS}_{2}$. Furthermore, the UV-Vis absorption (monitored along the surface normal) was significantly increased in the NaT3 film on $\|-\mathrm{MoS}_{2}$ as a result of the molecules adopting face-on orientation.

These results, in addition to highlighting the capabilities of in situ GIXRD, manifest the importance of film-substrate interactions on the growth and structure formation in vacuum deposited organic thin films. This is important in organic transistors, where the charge transport takes place in plane within the first few MLs, and where the transport may be limited by grain boundaries, as well as in OLED/OPV applications with out-of-plane charge transport. In future studies, it would be desirable to investigate the charge transport in situ to enable direct comparison with the here reported variations in unit cell packing and intergrain connectivity.

\section{ACKNOWLEDGMENTS}

The authors thank DESY (Hamburg, Germany) and SOLEIL (Saint-Aubin, France) for the provision of experimental facilities, Arne Lützen and Andreas Osadnik of the University of Bonn for providing NaTn powders, and Michael Winokur of the University of Wisconsin-Madison for discussions. The authors gratefully acknowledge the financial support of the Danish Agency for Science, Technology, and Innovation through the instrument center DanScatt, the Slovak Research and Development Agency (Project No. APVV-170352), the German Academic Exchange Service (DAAD), the Slovak Academy of Sciences (SAV), and the German Research Foundation (DFG). This work was performed during the implementation of the project Building-up Centre of Excellence for advanced materials application (ITMS Project No. 313021T081), supported by the Research and Innovation Operational Programme funded by the ERDF.
[1] Y. Wang, L. Sun, C. Wang, F. Yang, X. Ren, X. Zhang, H. Dong, and $\mathrm{W}$. Hu, Organic crystalline materials in flexible electronics, Chem. Soc. Rev. 48, 1492 (2019).

[2] P. R. Warren, J. F. M. Hardigree, A. E. Lauritzen, J. Nelson, and M. Riede, Tuning the ambipolar behaviour of organic field effect transistors via band engineering, AIP Adv. 9, 035202 (2019).

[3] J. Yuan, Y. Zhang, L. Zhou, G. Zhang, H.-L. Yip, T.-K. Lau, X. Lu, C. Zhu, H. Peng, P. A. Johnson, M. Leclerc, Y. Cao, J. Ulanski, Y. Li, and Y. Zou, Single-junction organic solar cell with over $15 \%$ efficiency using fused-ring acceptor with electron-deficient core, Joule 3, 1140 (2019).

[4] V. Jankus, P. Data, D. Graves, C. McGuinness, J. Santos, M. R. Bryce, F. B. Dias, and A. P. Monkman, Highly efficient TADF OLEDs: How the emitter-host interaction controls both the excited state species and electrical properties of the devices to achieve near $100 \%$ triplet harvesting and high efficiency, Adv. Funct. Mater. 24, 6178 (2014).

[5] Y. Liu, C. Li, Z. Ren, S. Yan, and M. R. Bryce, All-organic thermally activated delayed fluorescence materials for organic light-emitting diodes, Nat. Rev. Mater. 3, 18020 (2018).

[6] J. Lu, D. Liu, J. Zhou, Y. Chu, Y. Chen, X. Wu, and J. Huang, Porous organic field-effect transistors for enhanced chemical sensing performances, Adv. Funct. Mater. 27, 1700018 (2017).

[7] H. Li, W. Shi, J. Song, H.-J. Jang, J. Dailey, J. Yu, and H. E. Katz, Chemical and biomolecule sensing with organic fieldeffect transistors, Chem. Rev. 119, 3 (2018).

[8] S. Fratini, M. Nikolka, A. Salleo, G. Schweicher, and H. Sirringhaus, Charge transport in high-mobility conjugated poly- mers and molecular semiconductors, Nat. Mater. 19, 491 (2020).

[9] M. Brinkmann, L. Hartmann, N. Kayunkid, and D. Djurado, Understanding the Structure and Crystallization of Regioregular Poly (3-hexylthiophene) from the Perspective of Epitaxy, in P3HT Revisited From Molecular Scale to Solar Cell Devices. Advances in Polymer Science, Vol. 265, edited by S. Ludwigs (Springer, Berlin, 2014), pp. 83-106.

[10] R. Hildner, A. Köhler, P. Müller-Buschbaum, F. Panzer, and M. Thelakkat, $\pi$-conjugated donor polymers: Structure formation and morphology in solution, bulk and photovoltaic blends, Adv. Energy Mater. 7, 1700314 (2017).

[11] H. Sirringhaus, R. J. Wilson, R. H. Friend, M. Inbasekaran, W. Wu, E. P. Woo, M. Grell, and D. D. C. Bradley, Mobility enhancement in conjugated polymer field-effect transistors through chain alignment in a liquid-crystalline phase, Appl. Phys. Lett. 77, 406 (2000).

[12] J.-C. Ribierre, T. Tanaka, L. Zhao, Y. Yokota, S. Matsumoto, D. Hashizume, K. Takaishi, T. Muto, B. Heinrich, S. Méry, F. Mathevet, T. Matsushima, M. Uchiyama, C. Adachi, and T. Aoyama, Simultaneous edge-on to face-on reorientation and $1 \mathrm{~d}$ alignment of small $\pi$-conjugated molecules using room-temperature mechanical rubbing, Adv. Funct. Mater. 28, 1707038 (2018).

[13] S. E. Fritz, S. M. Martin, C. D. Frisbie, M. D. Ward, and M. F. Toney, Structural characterization of a pentacene monolayer on an amorphous $\mathrm{SiO}_{2}$ substrate with grazing incidence x-ray diffraction, J. Am. Chem. Soc. 126, 4084 (2004). 
[14] E. M. Mannebach, J. W. Spalenka, P. S. Johnson, Z. Cai, F. J. Himpsel, and P. G. Evans, High hole mobility and thicknessdependent crystal structure in $\alpha$, $\omega$-dihexylsexithiophene single-monolayer field-effect transistors, Adv. Funct. Mater. 23, 554 (2012).

[15] A. O. F. Jones, B. Chattopadhyay, Y. H. Geerts, and R. Resel, Substrate-induced and thin-film phases: Polymorphism of organic materials on surfaces, Adv. Funct. Mater. 26, 2233 (2016).

[16] L. Pithan, D. Nabok, C. Cocchi, P. Beyer, G. Duva, J. Simbrunner, J. Rawle, C. Nicklin, P. Schäfer, C. Draxl, F. Schreiber, and S. Kowarik, Molecular structure of the substrateinduced thin-film phase of tetracene, J. Chem. Phys. 149, 144701 (2018).

[17] C. E. Mauldin, K. Puntambekar, A. R. Murphy, F. Liao, V. Subramanian, J. M. J. Fréchet, D. M. DeLongchamp, D. A. Fischer, and M. F. Toney, Solution-processable $\alpha, \omega$-distyryl oligothiophene semiconductors with enhanced environmental stability, Chem. Mater. 21, 1927 (2009).

[18] A. Hexemer and P. Müller-Buschbaum, Advanced grazingincidence techniques for modern soft-matter materials analysis, IUCrJ 2, 106 (2015).

[19] D. M. DeLongchamp, Y. Jung, D. A. Fischer, E. K. Lin, P. Chang, V. Subramanian, A. R. Murphy, and J. M. J. Fréchet, Correlating molecular design to microstructure in thermally convertible oligothiophenes: The effect of branched versus linear end groups, J. Phys. Chem. B 110, 10645 (2006).

[20] S. Kowarik, A. Gerlach, and F. Schreiber, Organic molecular beam deposition: Fundamentals, growth dynamics, and in situ studies, J. Phys.: Condens. Matter 20, 184005 (2008).

[21] C. Lorch, K. Broch, V. Belova, G. Duva, A. Hinderhofer, A. Gerlach, M. Jankowski, and F. Schreiber, Growth and annealing kinetics of $\alpha$-sexithiophene and fullerene $\mathrm{C}_{60}$ mixed films, J. Appl. Crystallogr. 49, 1266 (2016).

[22] L. J. Richter, D. M. DeLongchamp, and A. Amassian, Morphology development in solution-processed functional organic blend films: An in situ viewpoint, Chem. Rev. 117, 6332 (2017).

[23] T. L. Derrien, A. E. Lauritzen, P. Kaienburg, J. F. M. Hardigree, C. Nicklin, and M. Riede, In situ observations of the growth mode of vacuum-deposited $\alpha$-sexithiophene, J. Phys. Chem. C 124, 11863 (2020).

[24] R. Zimmerleiter, M. Hohage, and L. Sun, Real-time in situ fluorescence study of $\alpha-6 \mathrm{~T}$ thin film growth on muscovite mica, Phys. Rev. Materials 3, 083402 (2019).

[25] M. Ando, M. Yoneya, T. B. Kehoe, H. Ishii, T. Minakata, M. Kawasaki, C. M. Duffy, R. Phillips, and H. Sirringhaus, Disorder and localization dynamics in polymorphs of the molecular semiconductor pentacene probed by in situ microraman spectroscopy and molecular dynamics simulations, Phys. Rev. Materials 3, 025601 (2019).

[26] L. Zhang, X. Fu, M. Hohage, P. Zeppenfeld, and L. D. Sun, Growth of pentacene on $\alpha-\mathrm{Al}_{2} \mathrm{O}_{3}$ (0001) studied by in situ optical spectroscopy, Phys. Rev. Materials 1, 043401 (2017).

[27] A. J. Fleming, F. P. Netzer, and M. G. Ramsey, Nucleation and $3 D$ growth of para-sexiphenyl nanostructures from an oriented $2 D$ liquid layer investigated by photoemission electron microscopy, J. Phys.: Condens. Matter 21, 445003 (2009).

[28] O. Panova, C. Ophus, C. J. Takacs, K. C. Bustillo, L. Balhorn, A. Salleo, N. Balsara, and A. M. Minor, Diffraction imaging of nanocrystalline structures in organic semiconductor molecular thin films, Nat. Mater. 18, 860 (2019).
[29] A. Sarbu, P. Hermet, D. Maurin, D. Djurado, L. Biniek, M. Diebold, J.-L. Bantignies, P. Mésini, and M. Brinkmann, Supramolecular organization of a $\mathrm{H}$-bonded perylene bisimide organogelator determined by transmission electron microscopy, grazing incidence $\mathrm{x}$-ray diffraction and polarized infra-red spectroscopy, Phys. Chem. Chem. Phys. 19, 32514 (2017).

[30] M. Surin, P. Sonar, A. C. Grimsdale, K. Müllen, S. D. Feyter, S. Habuchi, S. Sarzi, E. Braeken, A. V. Heyen, M. Van der Auweraer, F. C. D. Schryver, M. Cavallini, J.-F. Moulin, F. Biscarini, C. Femoni, R. Lazzaroni, and P. Leclère, Solid-state assemblies and optical properties of conjugated oligomers combining fluorene and thiophene units, J. Mater. Chem. 17, 728 (2007).

[31] H. Tian, J. Shi, B. He, N. Hu, S. Dong, D. Yan, J. Zhang, Y. Geng, and F. Wang, Naphthyl and thionaphthyl end-capped oligothiophenes as organic semiconductors: Effect of chain length and end-capping groups, Adv. Funct. Mater. 17, 1940 (2007).

[32] M. J. Winokur, M. K. Huss-Hansen, A. E. Lauritzen, M. Torkkeli, J. Kjelstrup-Hansen, and M. Knaapila, Modeling of grazing-incidence $\mathrm{x}$-ray diffraction from naphthyl endcapped oligothiophenes in organic field-effect transistors, Cryst. Growth Des. 20, 3968 (2020).

[33] M. K. Huss-Hansen, M. Hodas, N. Mrkyvkova, J. Hagara, B. B. E. Jensen, A. Osadnik, A. Lützen, E. Majková, P. Siffalovic, F. Schreiber, L. Tavares, J. Kjelstrup-Hansen, and M. Knaapila, Surface-controlled crystal alignment of naphthyl endcapped oligothiophene on graphene: Thin-film growth studied by in situ X-ray diffraction, Langmuir 36, 1898 (2020).

[34] M. K. Huss-Hansen, A. E. Lauritzen, O. Bikondoa, M. Torkkeli, L. Tavares, M. Knaapila, and J. Kjelstrup-Hansen, Structural stability of naphthyl end-capped oligothiophenes in organic field-effect transistors measured by grazing-incidence x-ray diffraction in operando, Org. Electron. 49, 375 (2017).

[35] J. Linnet, A. R. Walther, O. Albrektsen, L. Tavares, R. L. Eriksen, P. B. W. Jensen, A. Osadnik, S. Hassing, A. Lützen, and J. Kjelstrup-Hansen, Enhanced photoresponsivity in organic field effect transistors by silver nanoparticles, Org. Electron. 46, 270 (2017).

[36] A. E. Lauritzen, M. Torkkeli, O. Bikondoa, J. Linnet, L. Tavares, J. Kjelstrup-Hansen, and M. Knaapila, Structural evaluation of 5, 5' -bis(naphth-2-yl)-2, 2' -bithiophene in organic field-effect transistors with n-octadecyltrichlorosilane coated $\mathrm{SiO}_{2}$ gate dielectric, Langmuir 34, 6727 (2018).

[37] F. Balzer, M. Schiek, A. Osadnik, I. Wallmann, J. Parisi, H.-G. Rubahn, and A. Lützen, Substrate steered crystallization of naphthyl end-capped oligothiophenes into nanofibers: The influence of methoxy-functionalization, Phys. Chem. Chem. Phys. 16, 5747 (2014).

[38] J. Hagara, N. Mrkyvkova, P. Nádaždy, M. Hodas, M. Bodík, M. Jergel, E. Majková, K. Tokár, P. Hutár, M. Sojková, A. Chumakov, O. Konovalov, P. Pandit, S. Roth, A. Hinderhofer, M. Hulman, P. Siffalovic, and F. Schreiber, Reorientation of $\pi$-conjugated molecules on few-layer $\mathrm{MoS}_{2}$ films, Phys. Chem. Chem. Phys. 22, 3097 (2020)

[39] M. Sojková, P. Siffalovic, O. Babchenko, G. Vanko, E. Dobročka, J. Hagara, N. Mrkyvkova, E. Majková, T. Ižák, A. Kromka, and M. Hulman, Carbide-free one-zone sulfurization method grows thin $\mathrm{MoS}_{2}$ layers on polycrystalline CVD diamond, Sci. Rep. 9, 2001 (2019). 
[40] A. Buffet, A. Rothkirch, R. Döhrmann, V. Körstgens, M. M. A. Kashem, J. Perlich, G. Herzog, M. Schwartzkopf, R. Gehrke, P. Müller-Buschbaum, and S. V. Roth, P03, the microfocus and nanofocus $\mathrm{x}$-ray scattering (MiNaXS) beamline of the PETRA III storage ring: The microfocus endstation, J. Synchrotron Radiat. 19, 647 (2012).

[41] K. A. Ritley, B. Krause, F. Schreiber, and H. Dosch, A portable ultrahigh vacuum organic molecular beam deposition system for in situ x-ray diffraction measurements, Rev. Sci. Instrum. 72, 1453 (2001).

[42] M. Basham, J. Filik, M. T. Wharmby, P. C. Y. Chang, B. E. Kassaby, M. Gerring, J. Aishima, K. Levik, B. C. A. Pulford, I. Sikharulidze, D. Sneddon, M. Webber, S. S. Dhesi, F. Maccherozzi, O. Svensson, S. Brockhauser, G. Náray, and A. W. Ashton, Data analysis WorkbeNch(DAWN), J. Synchrotron Radiat. 22, 853 (2015).

[43] J. A. Merlo, C. R. Newman, C. P. Gerlach, T. W. Kelley, D. V. Muyres, S. E. Fritz, M. F. Toney, and C. D. Frisbie, p-channel organic semiconductors based on hybrid acenethiophene molecules for thin-film transistor applications, J. Am. Chem. Soc. 127, 3997 (2005).

[44] D.-M. Smilgies, Scherrer grain-size analysis adapted to grazing-incidence scattering with area detectors, J. Appl. Crystallogr. 42, 1030 (2009).

[45] D. Nečas and P. Klapetek, Gwyddion: An open-source software for SPM data analysis, Cent. Eur. J. Phys. 10, 181 (2012).

[46] D. Kong, H. Wang, J. J. Cha, M. Pasta, K. J. Koski, J. Yao, and Y. Cui, Synthesis of $\mathrm{MoS}_{2}$ and $\mathrm{MoSe}_{2}$ films with vertically aligned layers, Nano Lett. 13, 1341 (2013).

[47] J. L. Baker, L. H. Jimison, S. Mannsfeld, S. Volkman, S. Yin, V. Subramanian, A. Salleo, A. P. Alivisatos, and M. F. Toney, Quantification of thin film crystallographic orientation using x-ray diffraction with an area detector, Langmuir 26, 9146 (2010).

[48] M. K. Huss-Hansen, M. Hansteen, J. Linnet, A. R. Walther, J. Kjelstrup-Hansen, and M. Knaapila, Structural basis for a naphthyl end-capped oligothiophene with embedded metallic nanoparticles for organic field-effect transistors, Appl. Phys. Lett. 113, 251903 (2018).

[49] See Supplemental Material at http://link.aps.org/supplemental/ 10.1103/PhysRevMaterials.5.053402 for additional data figures.

[50] D. Bowen and B. Tanner, High Resolution X-Ray Diffractometry And Topography (CRC Press, Boca Raton, FL, 1998).

[51] S. Kowarik, A. Gerlach, S. Sellner, F. Schreiber, L. Cavalcanti, and O. Konovalov, Real-Time Observation of Structural and Orientational Transitions During Growth of Organic Thin Films, Phys. Rev. Lett. 96, 125504 (2006).

[52] S. K. Sinha, E. B. Sirota, S. Garoff, and H. B. Stanley, X-ray and neutron scattering from rough surfaces, Phys. Rev. B 38, 2297 (1988)

[53] S. Bommel, N. Kleppmann, C. Weber, H. Spranger, P. Schäfer, J. Novak, S. V. Roth, F. Schreiber, S. H. L. Klapp, and S.
Kowarik, Unravelling the multilayer growth of the fullerene $\mathrm{C}_{60}$ in real time, Nat. Commun. 5, 5388 (2014).

[54] T. Watanabe, T. Koganezawa, M. Kikuchi, H. Muraoka, S. Ogawa, N. Yoshimoto, and I. Hirosawa, In situ characterization of the film coverage and the charge transport in the alkylatedorganic thin film transistor, Jpn. J. Appl. Phys. 57, 03EG14 (2018).

[55] J. Hagara, N. Mrkyvkova, L. Feriancová, M. Putala, P. Nádaždy, M. Hodas, A. Shaji, V. Nádaždy, M. K. Huss-Hansen, M. Knaapila, J. Hagenlocher, N. Rußegger, M. Zwadlo, L. Merten, M. Sojková, M. Hulman, A. Vlad, P. Pandit, S. V. Roth, M. Jergel et al., Novel highly substituted thiophene-based n-type organic semiconductor: Structural study, optical anisotropy and molecular control, CrystEngComm 22, 7095 (2020).

[56] C. Schünemann, D. Wynands, K.-J. Eichhorn, M. Stamm, K. Leo, and M. Riede, Evaluation and control of the orientation of small molecules for strongly absorbing organic thin films, J. Phys. Chem. C 117, 11600 (2013).

[57] N. Shioya, R. Murdey, K. Nakao, H. Yoshida, T. Koganezawa, K. Eda, T. Shimoaka, and T. Hasegawa, Alternative face-on thin film structure of pentacene, Sci. Rep. 9, 579 (2019).

[58] K. Yamada, M. Suzuki, T. Suenobu, and K. Nakayama, High vertical carrier mobilities of organic semiconductors due to a deposited laid-down herringbone structure induced by a reduced graphene oxide template, ACS Appl. Mater. Interfaces 12, 9489 (2020).

[59] H. Proehl, T. Dienel, R. Nitsche, and T. Fritz, Formation of Solid-State Excitons in Ultrathin Crystalline Films of PTCDA: From Single Molecules to Molecular Stacks, Phys. Rev. Lett. 93, 097403 (2004)

[60] U. Heinemeyer, K. Broch, A. Hinderhofer, M. Kytka, R. Scholz, A. Gerlach, and F. Schreiber, Real-Time Changes in the Optical Spectrum of Organic Semiconducting Films and Their Thickness Regimes During Growth, Phys. Rev. Lett. 104, 257401 (2010).

[61] S. Kowarik, A. Gerlach, S. Sellner, L. Cavalcanti, and F. Schreiber, Dewetting of an organic semiconductor thin film observed in real-time, Adv. Eng. Mater. 11, 291 (2009).

[62] A. C. Dürr, F. Schreiber, K. A. Ritley, V. Kruppa, J. Krug, H. Dosch, and B. Struth, Rapid Roughening in Thin Film Growth of an Organic Semiconductor (Diindenoperylene), Phys. Rev. Lett. 90, 016104 (2003).

[63] B. Krause, F. Schreiber, H. Dosch, A. Pimpinelli, and O. H Seeck, Temperature dependence of the $2 \mathrm{~d}-3 \mathrm{~d}$ transition in the growth of PTCDA on ag(111): A real-time x-ray and kinetic monte carlo study, Europhys. Lett. 65, 372 (2004).

[64] L. Kankate, F. Balzer, H. Niehus, and H.-G. Rubahn, Organic nanofibers from thiophene oligomers, Thin Solid Films $\mathbf{5 1 8}$ 130 (2009).

[65] G. Koller, S. Berkebile, J. R. Krenn, G. Tzvetkov, G. Hlawacek, O. Lengyel, F. P. Netzer, C. Teichert, R. Resel, and M. G. Ramsey, Oriented sexiphenyl single crystal nanoneedles on $\mathrm{TiO}_{2}$ (110), Adv. Mater. 16, 2159 (2004). 